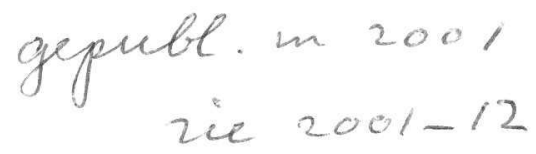

\title{
MIMO Closed-loop Identification of a MSW Incinerator
}

\author{
M. Leskens ${ }^{\S}{ }^{*}$, L.B.M. van Kessel ${ }^{\S}$ and P.M.J. Van den Hof $\sharp$ \\ $\S$ TNO Environment, Energy and Process Innovation \\ Laan van Westenenk 501, P.O. Box 342, 7300 AH Apeldoorn, The Netherlands. \\ \# Signals, Systems and Control Group \\ Department of Applied Physics, Delft University of Technology, Lorentzweg 1, 2628 CJ Delft, \\ The Netherlands
}

\begin{abstract}
In this paper the application of a specific system identification procedure to a municipal solid waste (MSW) incinerator is discussed. This procedure is a combination of, on the one hand, a particular closed-loop identification method called the two stage method and, on the other hand, the approach of high order MIMO ARX model estimation followed by model reduction. MIMO ARX model estimation is performed by means of a, so called, multiple data set identification method, i.e. a method by means of which it is possible to estimate a model on the basis of several data sets instead of just one data set. Model reduction is applied to each transfer function of the resulting MIMO ARX model separately. It is shown that with the proposed identification procedure a model of the MSW incinerator is obtained which, according to system identification validation measures, is good. Using the estimated model, the influence of the disturbances on the identification and control of a MSW incinerator is discussed. Furthermore, the validation of a first-principles model of the MSW incineration process by means of the resulting low order SISO models is discussed. The results show that the proposed way of validating a first-principles model is a powerful tool for determining its quality.
\end{abstract}

Keywords. MIMO ARX model estimation, closed-loop identification, two stage method, multiple data set idsntification, model validation, municipal solid waste incineration

\section{Introduction}

Incineration of municipal solid waste is used to reduce the amount of waste and to produce energy. Mainly due to strict environmental regulations a MSW incinerator is a capital-intensive process of which the capital costs have to be regained from the profits obtained from the incineration of waste and the production of energy. Due to the ever becoming more stringent environmental regulations and the necessity to operate more and more efficiently, MSW incinerators are faced with a growing need for a more optimal operation and, as a result of that, optimization of the MSW incineration process.

\footnotetext{
* Author to whom correspondence should be adressed. Email: m.leskens@mep.tno.nl.
}

Optimal operation of a MSW incinerator is difficult due to the heavy disturbances acting on the process which are the result of the heavily fluctuating waste composition. Disturbance rejection is in fact the main goal of the control system of a MSW incinerator. Optimization of the MSW incineration process is difficult due to its complexity and lack of knowledge about the phenomena taking place in this process. As a result, during in particular the last decade, research has been carried out with the aim to model these phenomena. Results of this research are mainly static, theory based (i.e. non-empirical) mathematical descriptions (obtained, for example, by means of computational fluid dynamics modeling) of the phenomena taking place during MSW 
incineration. See, for example, Gort (1995), Goh et al. (1998) and Shin et al. (1999). The literature on the dynamic and/or experimental modeling of the MSW incineration process, on the other hand, is scarce. In particular, no literature exists about the modeling of the MSW incineration process by means of system identification.

Because of the scarcity of literature on the dynamic modeling of the MSW incineration process, a dynamic model of this process has been developed at TNO which is based both on first principles (introducing dynamics by means of conservation laws) and on the static modeling work of Gort (1995). This model, which is discussed in Van Kessel (2002), had to be validated, i.e. confronted with real-life data in order to assess its quality. As earlier results showed (Leskens et al. (1999)), validation of the dynamics of the MSW incineration process directly on the basis of the experimentally obtained data is very difficult due to the presence of heavy disturbances. In this paper another approach of validating the first-principles model is discussed consisting of an identification step followed by a step where the dynamics of the first-principles model is compared and adapted to the dynamics of the estimated model. With this approach it was thought to be possible to cancel out the influence of the disturbances on the validation of the first-principles model. The in- and output data to be used for the identification step were obtained from the MSW incinerator N.V. Huisvuilcentrale N-H (HVC) at Alkmaar, the Netherlands. The main part of this paper deals with the identification procedure that was used to estimate a MIMO model of this MSW incinerator. Also, a comparison is given between the resulting estimated model and the validated first-principles model (i.e. after it has been compared and adapted to the dynamics of the estimated model). Specific attention is given to two specific aspects that were encountered during identification: closed-loop system identification and, so called, multiple data set identification. The latter aspect refers to the situation that more than one data set is available for estimation.

This paper is organized as follows. First, in section 2, the MSW incinerator HVC is described. Section 3 is then devoted to the system identification techniques that were used to model the dynamics of this MSW incinerator. Specific attention is given to closed-loop identification, multiple data set identification and the identification procedure that was applied to the MSW incinerator. In section 4 results obtained with the application of the proposed identification procedure to the MSW incinerator HVC are discussed. First, the identification experiments that were performed at this MSW incinerator, aspects of the estimation and the way the validation of the first-principles model was performed are discussed. After that, the influence of the disturbances on the identification and control of a MSW incinerator is discussed. Also, a comparison is made between the dynamics of the estimated model and the dynamics of the validated first-principles model. Finally, in section 5 , the conclusions are given.

\section{The MSW incinerator HVC}

Municipal solid waste is collected from households and transported to the MSW incinerator, mostly by train or truck. There it is stored in a large bunker and mixed as much as possible by means of cranes to obtain a more homogeneous waste composition. Subsequently, the waste is transported by cranes into a large chute. See figure 1. At the bottom

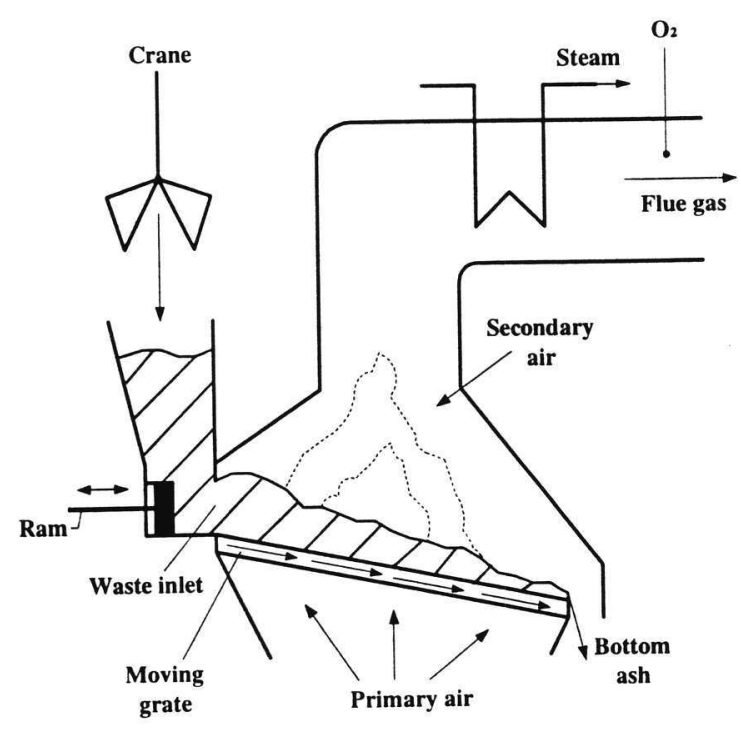

Fig. 1: The MSW incineration process.

of the chute, at the waste inlet, the waste is pushed onto a moving grate by a ram. The waste, the fuel of the combustion process, is incinerated while it is traveling on this grate. Air is added to the combustion process by means of two air flows: a primary and a secondary air flow. The primary air flow, delivering most of the oxygen, comes from beneath the grate, flowing through holes in this grate and, subsequently, through the solid waste bed. The secondary air is added to the combustion process through holes in the wall above the grate. The main function of this secondary air flow is to reduce the amount of unburnt gases coming from the waste bed. The combustion processes taking place in the solid waste layer are complex. Among these processes are (besides combustion) gasification and 
pyrolysis. In Gort (1995) and Goh et al. (1998) these processes in the waste layer are described both qualitatively and mathematically. A description of the phenomena taking place in the gas phase above the waste layer by means of computational fluid dynamics modeling can be found in Shin et al. (1999). The burnt gases and remaining unburnt gases enter a boiler delivering heat which is transformed into steam. Finally, the flue gas leaving the boiler is cleaned from residues that are not allowed to be fed to the surroundings. This cleaning part is not depicted in figure 1 but it contributes most to the size of a MSW incinerator due to the stringent environmental regulations. The amount of oxygen in the flue gas leaving the boiler is measured. It is an important variable to be controlled because it is not allowed to exceed certain limits due to requirements resulting from environmental considerations. Also the steam production is measured. It is an important variable to be controlled because it is a measure for the amount of energy that is produced by the incinerator. The remains of the solid waste on the grate, i.e. bottom ash, is disposed of or is used as, for example, raw material for asphalt.

The MSW incinerator HVC is one of the $11 \mathrm{MSW}$ incinerators currently present in the Netherlands. It was built in 1995 and has three lines (i.e. three furnaces as depicted in figure 1) each one of which has a throughput of approximately 18.5 tonnes of waste per hour per line by means of which approximately 63 tonnes of steam $\left(400{ }^{\circ} \mathrm{C}, 42 \mathrm{bar}\right)$ is produced per hour per line.

For the identification of the dynamics of the MSW incinerator HVC, the waste inlet flow, the speed of the grate, the primary air flow and the secondary air flow were chosen as input signals and the steam production and the amount of oxygen in the flue gas leaving the boiler were chosen as output signals. As a result, the MSW incinerator HVC can be modeled from an identification point of view as depicted in figure 2. As indicated in this figure, the MSW incineration process suffers from disturbances. As mentioned before, these are caused by the (non-measurable) fluctuation of the waste composition. This fluctuation causes a fluctuation in the density and, in particular, the calorific value of the waste which both have a large influence on the outputs to be controlled, i.e. the steam production and the oxygen concentration in the flue gas leaving the boiler.

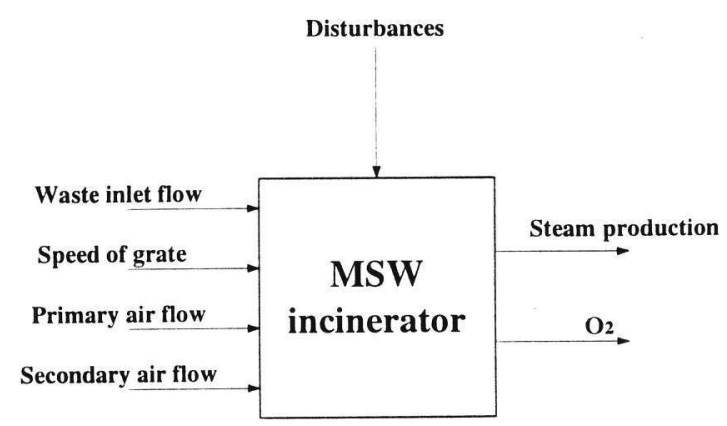

Fig. 2: The MSW incinerator from an identification (and control) point of view.

\section{System identification}

\subsection{Preliminaries}

\subsubsection{Prediction error method and MIMO ARX model structure}

For the identification of the dynamics of the MSW incinerator HVC the prediction error approach was followed. Following Ljung (1999), the data generating system is supposed to be given by the following multiple input multiple output (MIMO) linear time invariant (LTI) system:

$$
y(t)=G_{o}(q) u(t)+H_{o}(q) e(t)
$$

Here, $y(t)$ is a $p$-dimensional column vector consisting of output signals at time instant $t, u(t)$ is a $m$-dimensional column vector consisting of input signals, $e(t)$ is a $p$-dimensional column vector consisting of white noise signals, $G_{o}(q)$ an LTI system represented by its transfer function matrix in the forward-shift-operator $q$ and $H_{o}(q)$ a stable and stably invertible LTI system representing the noise system. A corresponding model of the system (1) is represented by the transfer function matrices $G(q, \theta)$ and $H(q, \theta)$ which are parametrized by the unknown vector of parameters $\theta$. This set of transfer function matrices defines the one-step-ahead prediction error

$$
\varepsilon(t, \theta)=H(q, \theta)^{-1}[y(t)-G(q, \theta) u(t)]
$$

The transfer function matrices $G(q, \theta)$ and $H(q, \theta)$ can be parametrized in several ways. The dynamics of the MSW incinerator HVC was identified using the MIMO ARX model structure. This model structure is represented by the transfer function matrices $G(q, \theta)=A(q, \theta)^{-1} B(q, \theta)$ and $H(q, \theta)=A(q, \theta)^{-1}$ with $A(q, \theta)$ and $B(q, \theta)$ given by the matrix polynomials

$$
A(q, \theta)=I_{p}+A_{1} q^{-1}+\ldots . .+A_{n a} q^{-n a}
$$


and

$$
B(q, \theta)=B_{0}+B_{1} q^{-1}+\ldots . .+B_{n b} q^{-n b}
$$

with $I_{p}$ a unity matrix of dimension $p \times p$. The polynomial degrees of the ARX model structure were chosen equal to each other, i.e. $n a=n b=n$, and all matrices $A_{i}, i=1 \ldots n$ and $B_{j}, j=0 . . . n$ were fully parametrized. When using the ARX model structure, the prediction error (2) is given by the linear regression form

$$
\varepsilon(t, \theta)=A(q, \theta) y(t)-B(q, \theta) u(t)=y(t)-\varphi^{T}(t) \theta
$$

with $\varphi(t)$ the so called regression matrix consisting of in- and output data from the past up to time instant $t$. When given a data set $Z_{N}$ of length $N$, an estimate $\hat{\theta}_{N}$ of the vector of parameters is obtained by minimizing the least squares criterion $V_{N}(\theta)$ defined by the optimization problem

$$
\begin{aligned}
\hat{\theta}_{N} & =\arg \min _{\theta} V_{N}(\theta) \\
& =\arg \min _{\theta} \frac{1}{N} \sum_{t=1}^{N} \varepsilon^{T}(t, \theta) \Lambda^{-1} \varepsilon(t, \theta)
\end{aligned}
$$

with $\Lambda$ a weighting matrix which usually is chosen equal to $I_{p}$ (the optimal value for this matrix would be the covariance matrix of the innovations $E e(t) e^{T}(t)$ with $E$ the expectation operator (Ljung (1999)) but this matrix is usually a priori unknown). The solution to the optimization problem (6) can analytically be obtained and is, for $\Lambda=I_{p}$, equal to

$$
\hat{\theta}_{N}=\left[\frac{1}{N} \sum_{t=1}^{N} \varphi(t) \varphi^{T}(t)\right]^{-1}\left[\frac{1}{N} \sum_{t=1}^{N} \varphi(t) y(t)\right]
$$

Because the solution can be obtained analytically, which is due to the fact that the prediction error is affine in the parameters, the solution can be obtained fastly which is the reason for its popularity when a model has to be estimated with many in- an outputs and thereby many parameters.

\subsubsection{QP formulation of the MIMO ARX model estimation problem}

The solution (7) to the optimization problem (6) with $\Lambda=I_{p}$ can also be obtained by solving the quadratic programming $(\mathrm{QP})$ problem

$$
\hat{\theta}_{N}=\arg \min _{\theta} 0.5 \cdot \theta^{T} H(t) \theta+g^{T}(t) \theta
$$

with the Hessian $H(t)$ equal to

$$
H(t)=\frac{1}{N} \sum_{t=1}^{N} \varphi(t) \varphi^{T}(t)
$$

and the gradient $g(t)$ equal to

$$
g(t)=-\frac{1}{N} \sum_{t=1}^{N} \varphi(t) y(t)
$$

Solving the unconstrained QP problem leads to the same solution for $\hat{\theta}_{N}$ as given by eqn. (7). However, the advantage of formulating the estimation problem as a QP problem is that equality and inequality constraints of the form $A \theta \leq b$ can be included easily into the optimization problem while software for solving such a constrained QP problem is widely available and a solution to the constrained QP problem can be obtained (almost) as equally fast as for the unconstrained case. The formulation of the estimation problem in the form of a QP problem is also useful when discussing multiple data set identification in section 3.2.2.

\subsection{Two practical problems and their solu- tions}

The general set-up given in the preceding section ignores some important practical problems that are often encountered when identifying an industrial process like the MSW incinerator HVC. Two of these problems will be discussed here. The first one refers to the estimation of a model on the basis of closed-loop data. The second problem refers to the estimation of a model on the basis of more than one data set.

\subsubsection{Closed-loop identification and the two stage method}

A typical situation that arises with the identification of an industrial process is that during the identification experiment(s) the controller is not allowed to be turned off due to, for example, safety or economic reasons. This was also the case for the identification of the MSW incinerator HVC for which the closed-loop configuration schematically is depicted in figure 3. As indicated in the figure, during the identification experiments the outputs of the controller were excited by (four) user-defined excitation signals $e x(t)$ while at the same time the (four) input signals $u(t)$ and (two) output signals $y(t)$ of the plant were measured.

As is known from literature (for example, Eek et al. (1996) and De Callafon (1998)), following the estimation procedure of section 3.1 directly, i.e. estimating the model directly on the basis of the inand output data $y(t)$ and $u(t)$, may result in a biased model. This bias grows with growing intensity level of the disturbances acting on the process to be identified (an illustrative example of this is given in De Callafon (1998)). A MSW incinerator suffers from disturbances with high power due 


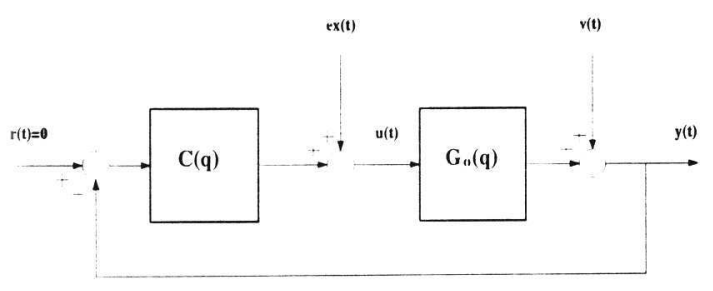

Fig. 3: Closed-loop (identification) configuration at the $M S W$ incinerator HVC: ex $(t)=$ excitation signals, $r(t)=$ reference signals, $u(t)=$ input signals, $G_{o}(q)=$ (true) process transfer function matrix, $v(t)=$ disturbances, $C(q)=$ controller transfer function matrix, $y(t)=$ output signals.

to the variation in waste composition. Therefore, a model of this process that is estimated directly on the basis of the experimentally obtained in- and output data is most likely to be biased. In order to prevent this bias a specific closed-loop identification method called the two stage method (Van den Hof and Schrama (1993)) was used.

For the closed-loop configuration of figure 3 , the inand output signals are given by $(r(t)=0)$

$$
\begin{aligned}
y(t)= & {\left[I+G_{o}(q) C(q)\right]^{-1} G_{o}(q) \operatorname{ex}(t)+} \\
& {\left[I+G_{o}(q) C(q)\right]^{-1} v(t) }
\end{aligned}
$$

and

$$
\begin{aligned}
u(t)= & {\left[I+C(q) G_{o}(q)\right]^{-1} e x(t)-} \\
& {\left[I+C(q) G_{o}(q)\right]^{-1} C(q) v(t) }
\end{aligned}
$$

The cause for a biased model is the correlation between the inputs $u(t)$ and the disturbances $[I+$ $\left.G_{o}(q) C(q)\right]^{-1} v(t)$ acting on the outputs which is the result of the presence of also a term due to the disturbances $v(t)$ in the inputs. The basic idea of the two stage method is to remove this correlation by removing the disturbance term in the input, i.e. the term $\left[I+C(q) G_{o}(q)\right]^{-1} C(q) v(t)$ in eqn. (12), before estimating the process transfer function matrix. This is done by estimating the transfer function matrix $\left[I+C(q) G_{o}(q)\right]^{-1}$ between the excitation signals $e x(t)$ and the input signals $u(t)$ (see eqn. (12)) as a first step (the first stage of the two stage method). With the resulting estimate, which is denoted by $S(q, \hat{\theta})$ and which is also called the input sensitivity function, the input signals without disturbance contribution, $u_{e x}(t)$, is then obtained by simulation:

$$
u_{e x}(t)=S(q, \hat{\theta}) e x(t)
$$

If $S(q, \theta)$ is estimated correctly then $u_{e x}(t)=[I+$ $\left.C(q) G_{o}(q)\right]^{-1} e x(t)$ which is equal to $u(t)$ (see eqn.
(12)) without the disturbance contribution. At the second stage, the process transfer function matrix is then estimated as the transfer matrix between $u_{e x}(t)$ and $y(t)$. Due to the absence of the disturbance contribution in the input signals $u_{e x}(t)$, the second step reduces to an open-loop identification problem and a consistent estimate of the process transfer function matrix $G_{o}(q)$ can be obtained.

For a further discussion of the direct identification problem, the two stage method, and other closedloop identification issues one is referred to the literature, for example Van den Hof and Schrama (1993), Van den Hof (1998) and De Callafon (1998).

\subsubsection{Multiple data set identification}

A second problem that typically might be encountered with the identification of an industrial process is that, due to the experimental conditions, not one data set but several (shorter) data sets are obtained from the identification experiment(s) which one all would like to use for the estimation of the model. Typical causes are for example that a number of separate experiments have been carried out instead of one and/or that bad data segments have to be removed from the obtained data set(s). This also was the case for the identification of the MSW incinerator HVC where the bad data segments typically were caused by a low calorific value of the waste, resulting in a drift of the operating point. In order to deal with this problem a specific so called multiple data set identification method was used employing the ARX model structure.

Suppose that $r$ data sets are available for estimation. The basic idea behind multiple data set identification is that the estimate $\hat{\theta}$ is obtained by minimizing the sum of $r$ criteria each of which is a function of one of the $r$ data sets and the same parameter vector $\theta$ :

$$
\hat{\theta}=\arg \min _{\theta} V(\theta)=\arg \min _{\theta} V_{1}(\theta)+\ldots . .+V_{r}(\theta)
$$

When using the ARX model structure, the optimization problem (14) can be simplified to a QP optimization problem of the form of eqn. (8) (Leskens (1998)) with the Hessian now equal to

$$
H(t)=H_{1}(t)+\ldots . .+H_{r}(t)
$$

and the gradient equal to

$$
g(t)=g_{1}(t)+\ldots . .+g_{r}(t)
$$

where $H_{j}(t)$ and $g_{j}(t)$ are the Hessian resp. gradient corresponding to data set $j$ which for each data set separately and $\Lambda=I_{p}$ are given by eqn. (9) and (10). Some characteristics of this multiple data set identification method are: 
- The parameter vector $\hat{\theta}$ is equal to the weighted sum of the parameter vectors that would be obtained when they would be estimated on the basis of a single data set:

$\hat{\theta}=\left[H_{1}(t)+\ldots+H_{r}(t)\right]^{-1}\left[H_{1}(t) \hat{\theta}_{1}+\ldots+H_{r}(t) \hat{\theta}_{r}\right]$

with $\hat{\theta}_{j}, j=1 \ldots r$, the parameter vector that would be estimated when only data set $j$ would be used for estimation. The weighting "factor" of each parameter vector $\hat{\theta}_{j}$ is the Hessian corresponding to data set $j$.

- Data sets can be combined which are obtained with a completely different distribution of the power over the frequencies. For example, a data set which is obtained with a step input signal, and thereby with most of its power in the low frequencies, can be combined with a data set that is obtained with a (P)RBS input signal with a high switching probability and thereby most of its power in the high frequencies.

- The combination of data sets in the way described above allows for user-defined scalar weighting of the data sets. As a result, the dynamics embedded in a specific data set can be given more weight in the optimization. This is useful when certain data sets are thought to be more reliable representations of the process to be identified than others.

A further discussion on multiple data set identification using the ARX model structure can be found in Leskens (1998) and Ljung (1999) (where it is referred to as the merging of data sets). Multiple data set identification for the FIR and so called ORTFIR model structure case is discussed in Van Donkelaar (2000).

\subsection{Identification strategy}

The approach that was used to identify the dynamics of the MSW incinerator HVC is typically used for the identification of large scale multivariable industrial processes: high order ARX modeling followed by a model reduction step. See, for example, Zhu \& Backx (1993) where a number of advantages of this strategy are given. Among them is the fact that, by using the asymptotic theory for prediction error methods, error bounds can be obtained for the estimated model which can be used in a robust control design method. Also, the asymptotic theory provides an explicit formulation for the optimal input signal spectrum to be used during identification experiments. Moreover, the selection of the model structure is greatly simplified (note that a structural identification step and the usage of (pseudo- )canonical parameterizations is avoided). Other advantages are that the method is numerically reliable and the estimate can be obtained relatively fast, even for processes with many in- and outputs and for high orders.

A high order is chosen for the ARX model (in literature figures ranging from $n=10$ to $n=50$ are found) in order to capture all relevant dynamics of the process to be identified. A high order model, however, is often not desirable in case of control design (due to, for example, implementation or computational reasons) and therefore model reduction is applied. In case of the MSW incinerator HVC, model reduction was applied for another reason: to obtain a low order approximate realization of the high order ARX model which does not exhibit the 'noisy' responses of the latter model. Model reduction was applied to each transfer function of the high order MIMO ARX model separately resulting in a low order SISO model for each of these transfer functions. The main reason for aiming at more smooth responses was that this facilitated the comparison between the estimated model and the first-principles model. Model reduction was performed by means of the approximate realization method of Van Helmont et al. (1990). This method will not not be discussed here in detail. It is similar to the well known realization algorithm of Kung (1978) but is based on step response coefficients rather than pulse response coefficients.

Combining the approach of high order ARX model estimation and model reduction with the two stage method, the identification strategy used for the identification of the MSW incinerator HVC can now be summarized as follows:

1. Estimation of the input sensitivity function $S(q, \hat{\theta})$ of eqn. (13) by estimating a high order MIMO ARX model (first stage of the two stage method).

2. Simulation of the inputs $u_{e x}(t)$ using the estimated input sensitivity function.

3. Estimation of the transfer function matrix from $u_{e x}(t)$ to $y(t)$ by estimating a high order MIMO ARX model (second stage of the two stage method).

4. Obtaining (8) low order SISO models of the high order MIMO ARX model that was estimated at the previous step by applying the method of Van Helmont et al. (1990) to all (8) transfer functions of this high order ARX model.

The choice of the model degree $n(=n a=n b)$ of both ARX models was based on the out- 
put/simulation error criterion

$$
V_{O E, i, n}=\frac{1}{N} \sum_{t=1}^{N}\left(\hat{y}_{i, n}(t)-y_{i}(t)\right)^{2}
$$

This criterion was not used directly: first scaling was applied in order to prevent an unfair comparison due to differences in dimension between the outputs and, secondly, the scaled criteria were averaged over all outputs. That model degree was chosen that corresponded to the lowest value for the scaled and averaged output error criterion. This choice turned out not to be critical, i.e. the differences between the finally obtained models (after model reduction) were small.

\section{Results}

\subsection{Experiments, estimation, comparison} and adaptation

The approach that was followed during identification is based on the approach given in Backx and Damen (1989) where the identification is split up in several steps among which data acquisition, pre-treatment of the experimentally obtained data, model estimation and validation (of the estimated model). The identification experiments were performed in July and August of 1998. The operating point values for these experiments are given in table 1 . During the experiments, the outputs of the

\begin{tabular}{|l|l|ll|}
\hline & Variable & Value & \\
\hline Inputs & Waste inlet flow & 53.2 & {$[\%]$} \\
& Speed of grate & 79.9 & {$[\%]$} \\
& Primary air flow & 52028 & {$\left[\mathrm{Nm}^{3} / \mathrm{h}\right]$} \\
& Secondary air flow & 10580 & {$\left[\mathrm{Nm}^{3} / \mathrm{h}\right]$} \\
\hline Outputs & Steam production & 16.3 & {$[\mathrm{~kg} / \mathrm{s}]$} \\
& Oxygen & 5.7 & {$[\%]$} \\
\hline
\end{tabular}

Table 1: Operating point values of the MSW incinerator HVC during the identification experiments.

controller were excited by user-defined test signals $(e x(t)$ in figure 3$)$. At first, some short preliminary experiments were performed in order to determine the frequency contents of the $(\mathrm{P}) \mathrm{RBS}$ test signals to be used during the final data acquisition experiments. With these data acquisition experiments, a total of 5 data sets were obtained with a length of up to 25 hours. One of the data sets was used for validation only. After these data acquisition experiments, a number of pre-treatment steps were applied to the experimentally obtained data. These included subtraction of the means and scaling. After that, the identification strategy described in section 3.3 was applied. This resulted in a model degree of $n=23$ for the estimated input sensitivity function and $n=18$ for the estimated process transfer function. The estimated high order ARX models were validated by means of the auto-correlation functions of the residuals and the cross-correlation functions between the input signals and these residuals. These correlation functions were computed on the basis of the validation data set. Both a typical auto- and cross-correlation function are shown in figure 4 . As can be seen in
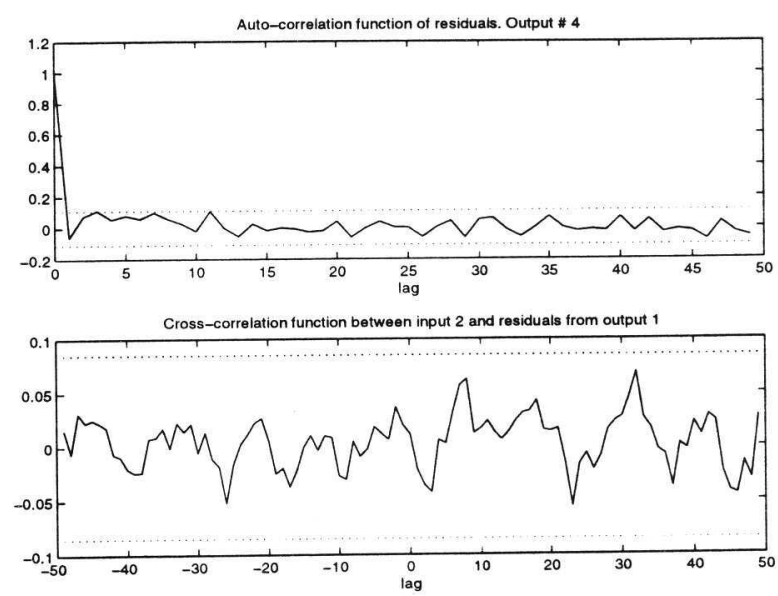

Fig. 4: An auto- and cross-correlation function for the estimated high order ARX model of the input sensitivity function, computed on the basis of the validation data set. Dotted lines $=99 \%$ confidence limits.

the figure, the correlation functions are good which implies that also the estimated model is good, at least for the dynamics incorporated in the validation data set. It also implies that the proposed identification strategy is capable of producing a good model of (an industrial process like) the MSW incinerator HVC. However, computed confidence intervals and personal knowledge of the incineration process indicated that the slowest dynamic behaviour, in particular the static behaviour, was incorporated not entirely well in this model. In order to improve the static and low frequency dynamical behaviour of the estimated model, a new estimation of the second high order ARX model was performed with now the static gains enforced on this model. These static gains were obtained from the first-principles model after this model was adapted statically to the firstprinciples model i.e. to the operating point values given in table 1. Enforcement of the static gains on the model to be estimated is common practice for the identification of industrial processes (see, for example, Ludlage et al. (1991)). One reason for the bad fit of the low frequency dynamic behaviour of an estimated model is that the obtained data set(s) 
often are relatively short compared to the dominant time constant of the process to be identified. Another reason is that the ARX model structure penalizes high-frequency misfit behaviour much more than low-frequency misfit behaviour (Ljung (1999)). The next step was the application of the approximate realization method of Van Helmont et al. (1990) to the resulting high order ARX model. This resulted in 8 low order SISO models which were subsequently used to simulate the dynamic behaviour of the MSW incinerator HVC. With the resulting responses the first-principles model was validated. This was performed by comparing the dynamic behaviour of both models and adapting manually a number of ( 6 physically related) parameters of the first-principles model until an agreement between the dynamics of both models was obtained which was (thought to be) as good as possible.

\subsection{The influence of the disturbances}

In this section, first, an idea will be given of the contribution of the disturbances in the experimentally obtained input signals. For that purpose, in figure 5 two of the four experimentally obtained input signals, i.e. primary and secondary air flow, are depicted together with their disturbance free counterparts; these were obtained by simulation using the estimated input sensitivity function as filter and the experimentally applied test signals as input signals of this filter, i.e. as $u_{e x}(t)=S(q, \hat{\theta}) \operatorname{ex}(t)$ (see eqn. 13). As one can see from this figure, the con-
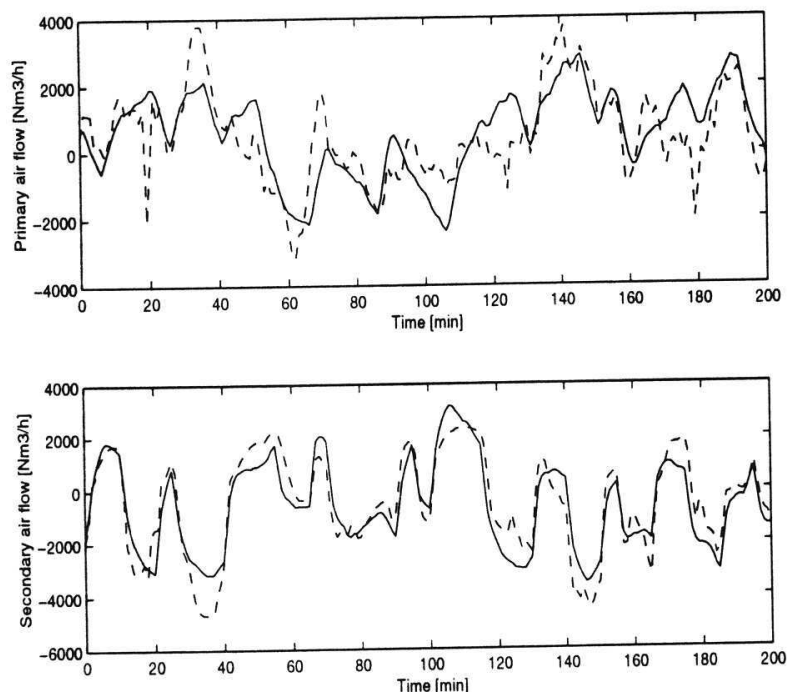

Fig. 5: Top figure: measured (- - ) and disturbance free, simulated, (-) primary air flow (see text). Bottom figure: measured (- -) and disturbance free, simulated, (-) secondary air flow. tribution of the disturbances in the inputs is significant. On the basis of the standard deviations of the experimentally obtained input signals and their corresponding disturbance free counterparts, the contribution of the disturbance part to the complete input signal varied for the four input signals from $3 \%$ (secondary air flow) to up to $30 \%$ (speed of grate) (20\% for the primary air flow). This indicates that the disturbance contribution in the input signals can be significant for a MSW incinerator and that the use of a specific closed-loop identification method like the two stage method is necessary to obtain a good model.

An idea of the contribution of the disturbances to the experimentally obtained output signals can be obtained from figure 6 . In this figure the two measured output signals steam production and oxygen concentration are depicted together with their disturbance free counter parts; in this case, these were obtained by simulation using the estimated process transfer as filter and the experimentally obtained input signals as input signals to this filter, i.e. as $G(q, \hat{\theta}) u(t)$ with $G(q, \hat{\theta})$ the estimated process transfer. (Note that if this transfer is equal to the real process transfer, i.e. $G(q, \hat{\theta})=G_{o}(q)$, the difference between the measured and simulated output signals is given by $\left.v(t)=y(t)-G_{o}(q) u(t)\right)$. From
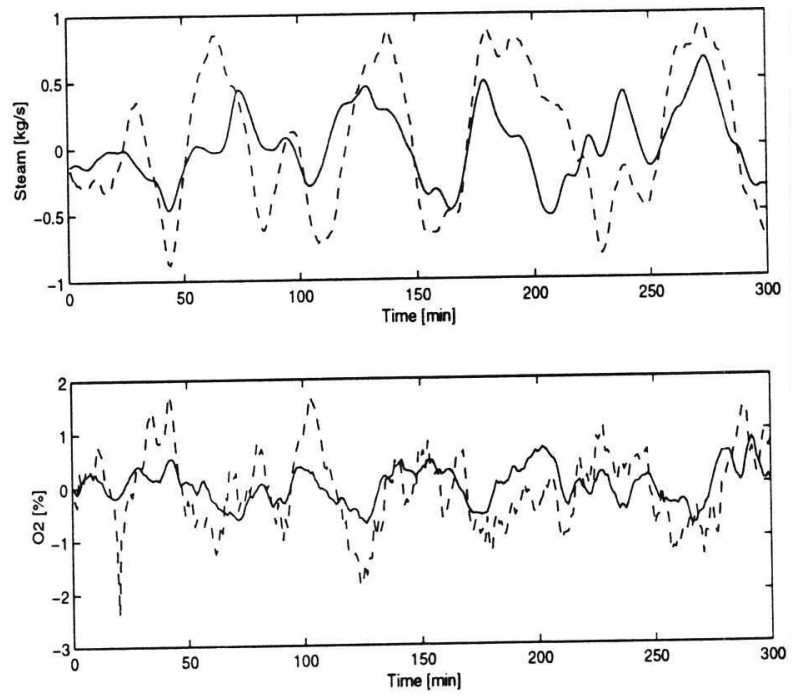

Fig. 6: Top figure: measured (- -) steam production and disturbance free, simulated, steam production (-) (see text). Bottom figure: measured (- -) oxygen concentration and disturbance free, simulated, oxygen concentration (-).

the figure it can be seen that the contribution of the disturbances is significant. This is confirmed by signal-to-noise ratios which were computed on the 
basis of computed standard deviations: the contribution of the disturbances to the steam production was $33 \%$ and even $49 \%$ for the oxygen concentration. The large fluctuations present in the steam production indicate that, in particular, also the temperature at the (inlet of) the steam system/boiler is subject to large fluctuations. This is an important variable from a control point of view as it is subject to an upper-limit due to considerations of corrosion. This thermal limit, in fact, also restricts the amount of waste throughput and, simultaneously, the steam production. As a result, this limit restricts the profitability of the plant because the amount of processed waste and produced steam are the main sources of income for a MSW incinerator. If one, however, could reduce the fluctuations in the temperature at the boiler, one could operate on average more closely to the thermal limit and, as $\mathrm{s}$ result, obtain a more profitable operation. This indicates the need for a controller for the MSW incineration process which is able to reduce the fluctuations in the process variables, in particular the temperature at the boiler, due to the variation in waste composition as much as possible.

\subsection{Comparison of the estimated and first- principles model}

In figures 7 to 10 simulated responses of the steam production and oxygen concentration obtained with the estimated and first-principles model are shown. These simulations were made with only one input signal excited at a time while the other input signals were kept constant (in case of the first-principles model) or zero (estimated model). The excited input signals are depicted in the lowest part of the figures and are equal to the (P)RBS test signals of the validation data set after having been filtered by the estimated input sensitivity function.

Figure 7 shows the simulated output signals when only the waste inlet flow is excited. One can see that both the simulated responses of the steam production and the predictions of the oxygen concentration coïncide very well. A similar good agreement between the simulated outputs of the estimated model and the first-principles model can be found in figures 8 and 9 . A less well agreement is found for the simulated outputs of the steam production as a function of the secondary air flow input signal (upper part of figure 10). No clear explanation can be given for this difference. Possible causes are unknown bad experimental conditions or improperly described dynamics in the first-principles model. Figure 10 also shows that the simulated responses of the oxygen concentration coïncide rather well.

The results show that it is possible to obtain a
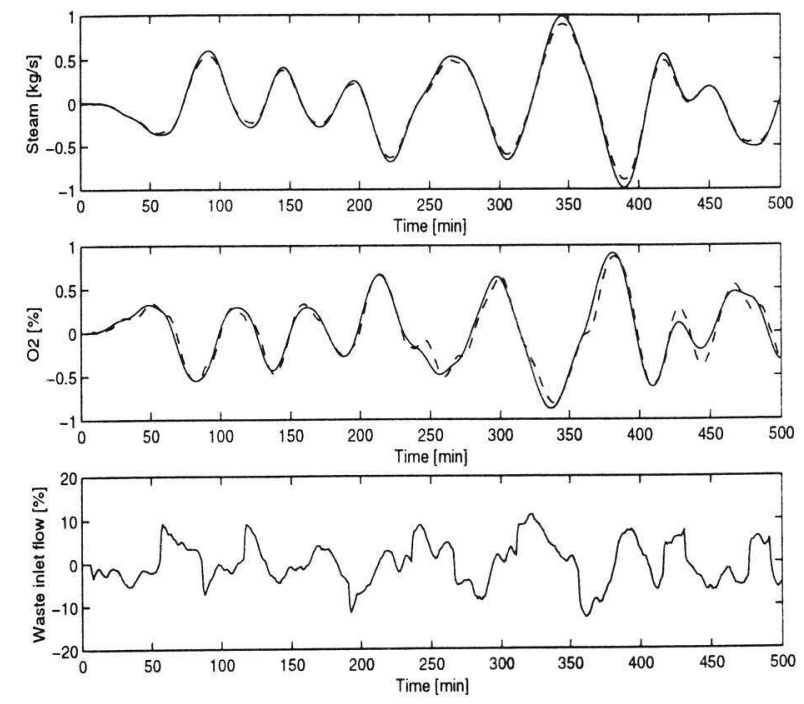

Fig. 7: Simulation of the steam production and oxygen concentration obtained with the estimated model (- -) and the first-principles model (-) when only the waste inlet flow (lowest part of figure) is excited.

clear picture about the quality of the first-principles model by validating it on the basis of an estimated model (which has (not) been (in)validated on the basis of pure system identification measures). In the case of the MSW incinerator HVC a clear picture was obtained of the dynamics that is incorporated well in the first-principles model. Of course one has to be cautious with this conclusion: theoretically, a lot (even an infinite number) of combinations of parameters of the first-principles model might lead to the same in- output behaviour (in identification terms this means that the model structure should be identifiable, i.e. two parameter sets/vectors should not lead to the same in- output behaviour. See Ljung (1999)). The choice of parameters to be adapted and the choice of their intervals was rather straightforward and a proper choice of these parameters and their intervals was thought to be made, giving rise to the conclusion stated just above.

\section{Conclusions}

In order to estimate a model of the MSW incinerator HVC a specific identification procedure was followed which was a combination of the two stage method and high order ARX modeling followed by a model reduction step: at both estimation steps of the two stage method a high order MIMO ARX model was estimated which was followed by a model reduction step applied to all (8) transfer functions of the second estimated high order ARX 

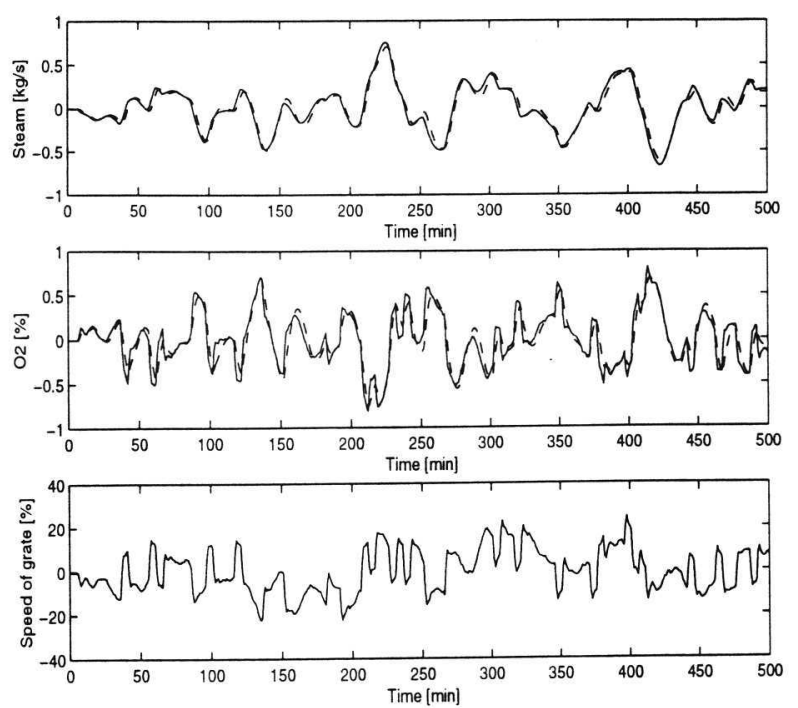

Fig. 8: Simulation of the steam production and oxygen concentration obtained with the estimated model (- -) and the first-principles model (-) when only the speed of the grate is excited.

model. For the estimation of the high order MIMO ARX models a specific so called multiple data set identification method was used. Also, static gains were enforced on the second estimated high order ARX model in order to improve the slowest dynamic behaviour of this model which was found to be not incorporated well in this model. The static gains were obtained from the first-principles model after this model was statically adapted to the MSW incinerator HVC. For the model reduction step the approximate realization method of Van Helmont et al. (1990) was used which employs step response coefficients of the system for which a low order approximate realization has to be obtained. With the applied identification procedure it turned out to be possible to estimate a good model of (an industrial process like) the MSW incinerator HVC. Using the estimated model and experimentally obtained data, it was shown that the influence of the disturbances acting on the MSW incinerator is large. These disturbances are of such high amplitude that the usage of a closed-loop identification method like the two stage method is necessary in order to obtain a good model of the process. The large influence of the disturbances also shows that a controller with good disturbance rejection properties is needed as these disturbances prohibit a higher throughput of waste and thereby a higher income for the MSW incinerator. The first-principles model was validated on the basis of the estimated model, i.e. compared
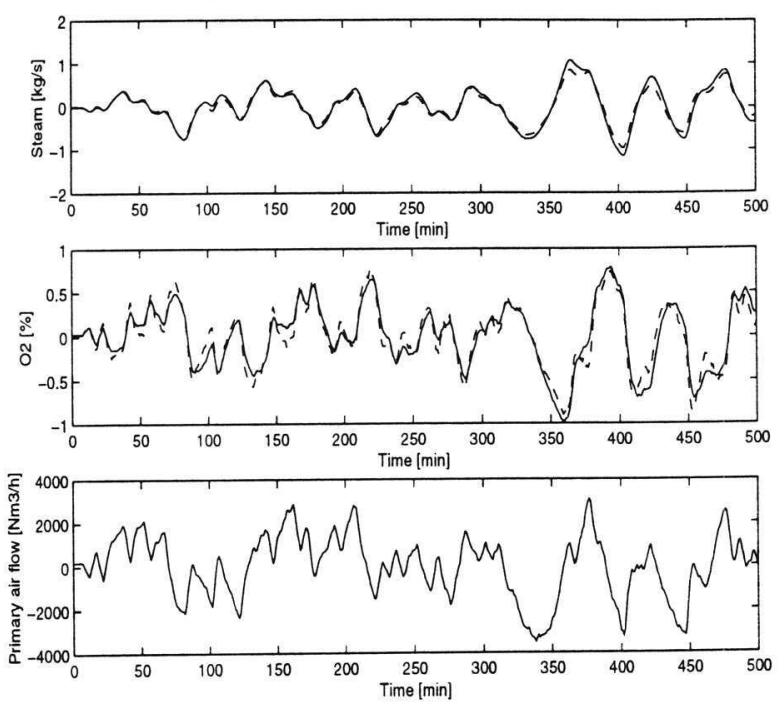

Fig. 9: Simulation of the steam production and oxygen concentration obtained with the estimated model (- -) and the first-principles model (-) when only the primary air flow is excited.

and adapted to the dynamics exhibited by the estimated model. This validation showed that most of the dynamics of the MSW incinerator HVC was incorporated well in the validated first-principles model. Comparing and adapting the dynamics of the first-principles model to the dynamics exhibited by an estimated model that has been validated by means of pure system identification validation measures proved to be a valuable tool for validation of the first-principles model.

\section{Acknowledgements}

The authors would like to thank N.V. Huisvuilcentrale N-H at Alkmaar, The Netherlands, for allowing the presentation of the results discussed in this paper.

\section{References}

Backx, A.C.P.M., \& Damen, A.A.H. (1989). Identification of industrial MIMO processes for fixed controllers. Part 1: General theory and practice, Journal A, Vol. 30, no.1, pp. 3-12

De Callafon, R. (1998). Feedback Oriented Identification for Enhanced and Robust Control: a fractional approach applied to a wafer stage, Dr. Dissertation, Delft University of Technology, Delft, Netherlands.

Eek, R.A., Both, J.A., \& Van den Hof, P.M.J. (1996). Closed-loop identification of a continuous crystallization process. AIChE Journal, Vol. 

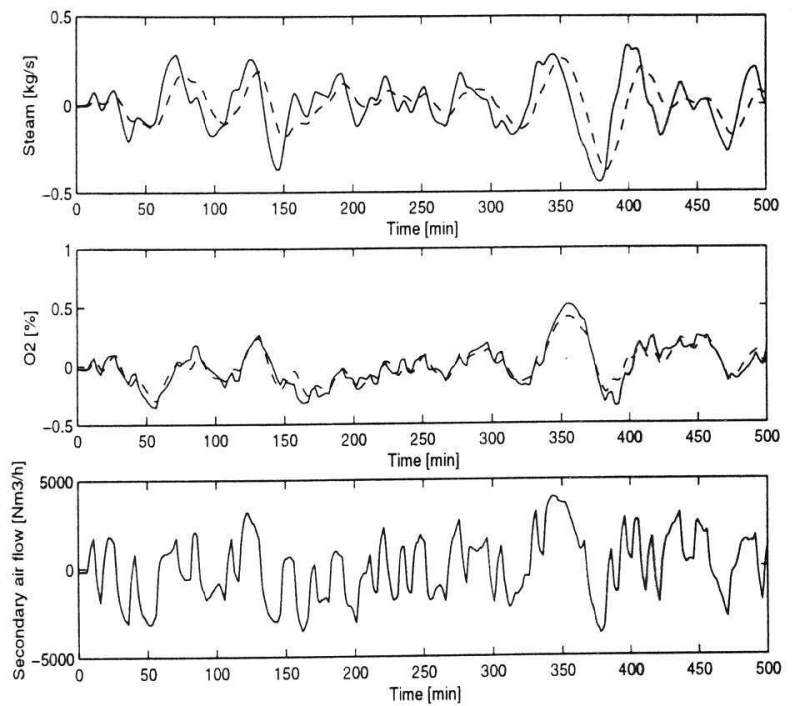

Fig. 10: Simulation of the steam production and oxygen concentration obtained with the estimated model (- -) and the firstprinciples model (-) when only the secondary air flow is excited.

42, No. 3.

Goh, Y.R., Siddall, R.G., Nasserzadeh, V., Zakaria, R., Swithenbank, J., Lawrence, D., Garrod, N., \& Jones, B. (1998). Mathematical modelling of the burning bed of a waste incinerator. Journal of the Institute of Energy, 71, pp. 110-118.

Gort, R. (1995). On the propagation of a reaction front in a packed bed: thermal conversion of municipal solid waste and biomass, Dr. Dissertation, Twente University of Technology, Enschede, Netherlands.

Kung, S. (1978). A new identification and model reduction algorithm via singular value decompositions, Proc. $12^{\text {th }}$ Asimolar Conf. Circuits Syst. and Computers, Pacific Grove, CA, pp. 705-714.

Leskens, M. (1998). Multivariable System Identification of a Fluidized Catalytic Cracking Unit. Report A-787, Mechanical Engineering Systems and Control Group, Faculty of Design, Engineering and Production, Delft University of Technology.

Leskens, M., Piatkiewitz, P.N.H.M., Van Kessel, L.B.M., \& Arendsen, A.R.J. (1999). Validatie van het TNO simulatiemodel van de afvalverbrandingsinstallatie in Emmenspitz (in Dutch), TNO report 99/155, TNO Milieu, Energie en Procesinnovatie.

Ljung, L. (1999). System Identification: Theory for the User. Second edition, Prentice-Hall, Upper Saddle River, NJ.

Ludlage, J., Oudbier, R., \& Backx, T. (1991). Use of A priori Knowledge of Static Gains in MIMO Process Identification. Proc. $9^{\text {th }}$ IFAC/IFORS Symp. Identification and System parameter estimation, Budapest, Hungary, pp. 1196-1201.

Shin, D., Choi J.H., Nasserzadeh V., Choi, S., \& Swithenbank J. (1999). Experimental measurements and computational modelling of a pilot scale incinerator. Journal of the Institute of Energy, 72, pp. 56-63.

Van den Hof, P.M.J., \& Schrama, R.J.P. (1993). An indirect method for transfer function estimation from closed loop Data. Automatica, 29, No. 6, pp. 1523-1527.

Van den Hof, P. (1998). Closed-loop issues in system identification. Annual Reviews in Control, 22, pp. 173-186.

Van Helmont, J.B., Van der Weiden, A.J.J., \& Anneveld, H. (1990). Design of an optimal controller for a coal fired Benson boiler based on a modified approximate realisation algorithm. In: $R$. Whalley (Ed.), Application of Multivariable System Techniques (AMST90). Elsevier Publ. Comp., London, pp. 313-320.

Van Donkelaar, E.T. (2000). Improvement of efficiency in system identification and model predictive control of industrial processes - A linear parametrization approach. Dr. Dissertation, Delft University of Technology, Delft, Netherlands.

Van Kessel, L.B.M. (2002). - . Dr. Dissertation, Eindhoven University of Technology, Eindhoven, Netherlands.

Van Kessel, L.B.M., Leskens, M., \& Verkerk, G.N. (1999). Het experimenteel vaststellen van het dynamisch gedrag van de afvalverbrandingsinstallatie HVC (in Dutch), TNO report 99/058, TNO Milieu, Energie en Procesinnovatie.

Zhu, Y., \& T. Backx (1993). Identification of Multivariable Industrial Processes for Simulation, Diagnosis and Control, Springer-Verlag. 